Case Report

\title{
Case Report of First Angiography-Based On-Line FFR Assessment during Coronary Catheterization
}

\author{
Ran Kornowski and Hana Vaknin-Assa \\ Cardiac Catheterization Laboratories, Cardiology Department, Rabin Medical Center, \\ Belinson Hospital Affiliated to the "Sackler" Faculty of Medicine, Tel Aviv University, Petah Tikva, Israel \\ Correspondence should be addressed to Ran Kornowski; ran.kornowski@gmail.com
}

Received 24 April 2017; Revised 19 June 2017; Accepted 3 July 2017; Published 1 August 2017

Academic Editor: Aiden Abidov

Copyright (C) 2017 Ran Kornowski and Hana Vaknin-Assa. This is an open access article distributed under the Creative Commons Attribution License, which permits unrestricted use, distribution, and reproduction in any medium, provided the original work is properly cited.

\begin{abstract}
Fractional flow reserve (FFR), an index of the hemodynamic severity of coronary stenoses, is derived from hyperemic pressure measurements and requires a pressure-monitoring guide wire and hyperemic stimulus. Although it has become the standard of reference for decision-making regarding coronary revascularization, the procedure remains underutilized due to its invasive nature. $\mathrm{FFR}_{\text {angio }}$ is a novel technology that uses the patient's hemodynamic data and routine angiograms to generate a complete threedimensional coronary tree, with color-coded display of the FFR values at each point along the vessels. After being proven to be as accurate as invasive FFR measurements in an off-line study, this case report presents the first on-line application of the system in the catheterization lab. Here too, a high concordance between $\mathrm{FFR}_{\text {angio }}$ and invasive FFR was observed. In light of the demonstrated capabilities of the $\mathrm{FFR}_{\text {angio }}$ system, it should emerge as an important tool for clinical decision-making regarding revascularization in patients with coronary artery disease.
\end{abstract}

\section{Introduction}

In patients with stable coronary disease, stenoses severity is assessed by pressure wire-based fractional flow reserve (FFR), a major and independent predictor of lesion-related coronary outcomes, which has become the standard of reference for decision-making regarding coronary revascularization. Yet, due to the invasive nature of the procedure FFR measurements remain underutilized $[1,2]$. Therefore, the ability to derive FFR values from routinely performed coronary angiography, without the need for a pressure guide wire or drug-induced hyperemic stimulus, could have an important impact on daily clinical practice.

Several image-based FFR techniques have been introduced in the past few years. Most are based on computational fluid dynamics (CFD), a method that uses numerical analysis and algorithms to calculate fluid flows within 3D structures. Their first validation tests were rather favorable but the computational complexity of CFD requires manual interaction, as well as a considerable processing time, which is problematic for "live" application in the catheterization lab. Additionally, the proposed tools usually allow for assessment of a specific lesion only within a chosen segment of the coronary artery [3-9].

FFR-angiography (FFR angio developed by CathWorks, Ra'anana, Israel) is a novel technology providing a threedimensional functional mapping of the coronaries. It is based on a rapid flow analysis of a dynamically derived lumped model which can assess FFR using routine angiograms. The accuracy of the $\mathrm{FFR}_{\text {angio }}$ system was tested and validated in a recent publication [10].

Following the acquisition of a normal angiogram, the user enters the mean aortic pressure. The coronary tree (right or left system) is reconstructed in 3D based on two or more conventional single-plane angiographic projections of the vessels, whereby epipolar ray tracing together with mathematical constraints enforcing the tree's structure is utilized. Next, the system scans the entire reconstructed tree in $3 \mathrm{D}$ and analyzes each branch as well as each bifurcation, looking for narrowed regions. Hyperemic flow ratio is derived from automatic resistance-based lumped mapping along the entire coronary tree. The FFR values at each point along the vessel are 

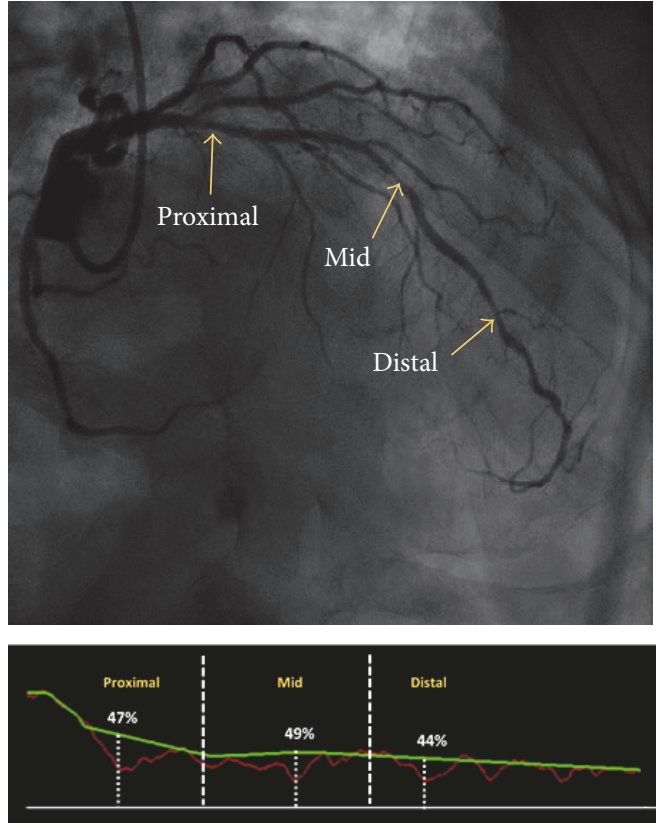

(a)
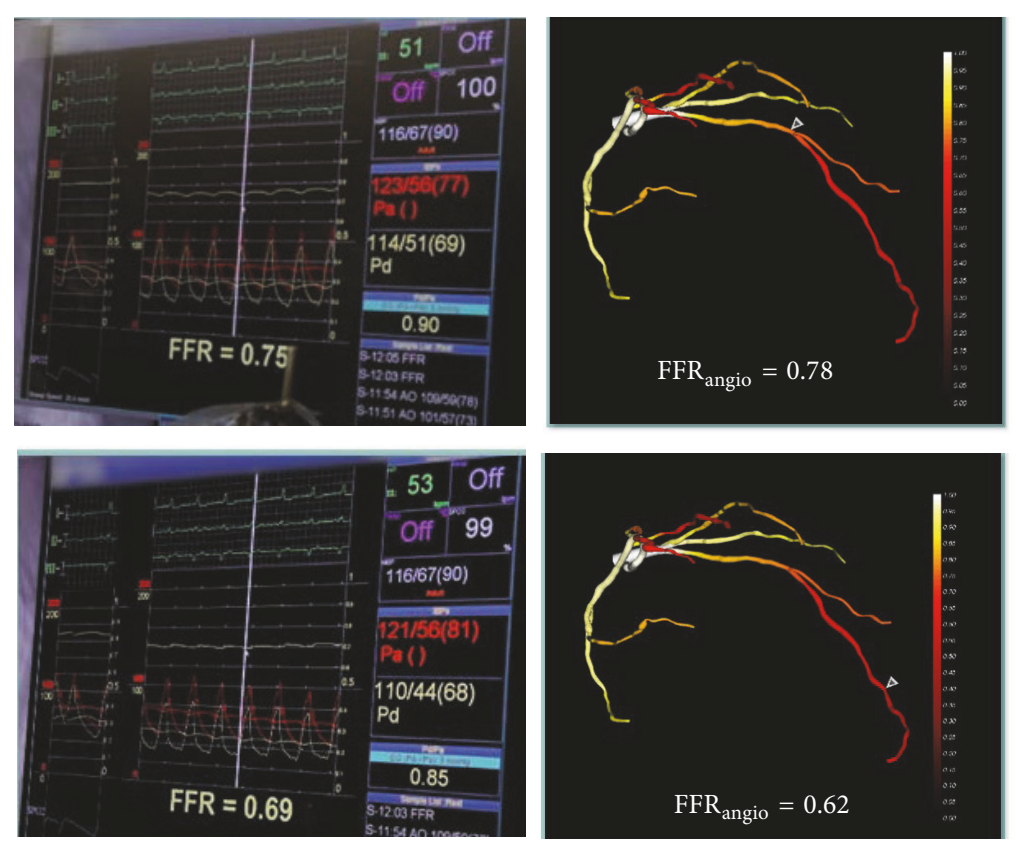

(b)

FIGURE 1: (a) (Top) LAD lesions (arrows indicate the proximal, mid, and distal lesions) and (bottom) corresponding percent diameter stenosis used for diagnostic FFR $_{\text {angio }}$ quantitative coronary angiography (QCA) calculation. (b) Invasive fractional flow reserve (FFR) measurements (left) of the middle (top) and distal (bottom) lesions and the corresponding values calculated by the $\mathrm{FFR}_{\text {angio }}$ system (right).

color-coded and superimposed on the 3D epicardial model and cut-off values of 0.80 identical to standard invasive FFR apply. FFR $_{\text {angio }}$ does not utilize pharmacologic druginduced hyperemia. This report describes the first "live" utilization of $\mathrm{FFR}_{\text {angio }}$ during an actual catheterization procedure.

\section{Case Presentation}

2.1. Clinical History. A 74-year-old man was referred to our hospital for diagnostic angiography. His clinical history included unstable angina, insulin-dependent diabetes mellitus, past smoking, dyslipidemia, and mild renal insufficiency. The patient underwent coronary catheterization the previous year due to stable angina; multiple moderate lesions were detected in the Left Anterior Descending (LAD) and a conservative treatment strategy was then recommended.

2.2. Case Description and Diagnosis. After 1 year, the patient was returned to the catheterization lab with NSTEMI. Diagnostic angiography revealed three consecutive discrete stenoses located in the proximal, middle, and distal segments of the LAD without evidence of diffuse disease (Figure 1(a)), and the patient was included as part of a 53-patient singlecenter study examining the accuracy of FFR angio when used on-line during the procedure. Based on visual estimation and the operator's decision, invasive FFR was performed for the middle and distal lesions only. Invasive fractional flow reserve (FFR) measurements were 0.75 and 0.69 , respectively (Figure 1(b)).
2.3. FFR angio. The FFR angio $_{\text {algorithm was applied in parallel }}$ to the invasive measurements. A $3 \mathrm{D}$ quantitative coronary angiography analysis (QCA) was derived and yielded diameter stenoses of $49 \%$ and $44 \%$ for the middle and distal LAD lesions, respectively. The system created a $3 \mathrm{D}$ image of the coronary tree showing the calculated $\mathrm{FFR}_{\text {angio }}$ values along the branches according to a color-coded scale. The calculated $\mathrm{FFR}_{\text {angio }}$ values at the exact locations of the invasive measurements (e.g., using a pressure wire) were 0.78 and 0.62 for the middle and distal lesions, respectively. Figure 1(b) presents the 3D QCA analysis as well as the invasive FFR and corresponding computed values obtained by the FFR ${ }_{\text {angio }}$ measurements for the middle and distal stenoses.

2.4. Poststenting FFR Evaluation. Following stenting of the middle and distal LAD lesions, invasive FFR measurement at the distal LAD segment was 0.74. Image-based FFR angio values at the distal LAD were calculated once again by using the posttreatment angiography, yielding a value of 0.71 , and compared to the invasive FFR value (Figure 2 ). As both FFR values remained significant $(<0.8)$ after stenting the middle and distal stenoses, the proximal LAD lesion was stented as well.

\section{Discussion}

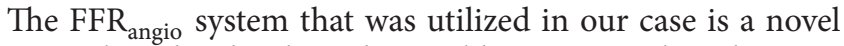
image-based technology that enables an image-based assessment of FFR, calculated from common angiograms reconstructed in 3D along with routinely measured hemodynamic data. Importantly, the FFR angio system, which is not based on 


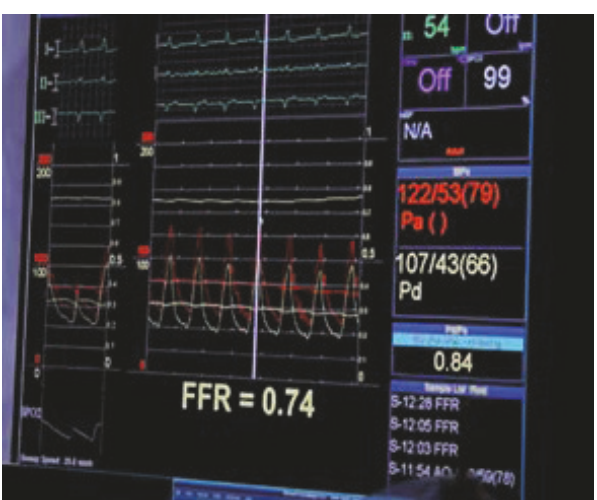

(a)

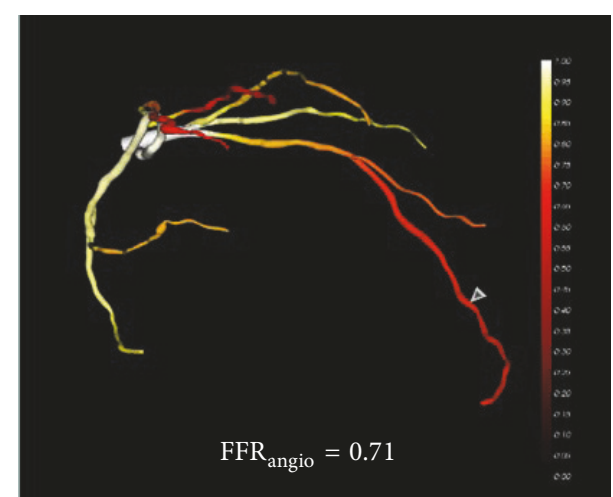

(b)

FIGURE 2: Posttreatment FFR (a) and $\mathrm{FFR}_{\text {angio }}$ (b) assessments at the distal LAD segment following stenting of the middle and distal lesions.

CFD, was designed to keep the pace of the catheterization procedure; that is, the time from image acquisition to display of the $\mathrm{FFR}_{\text {angio }}$ results is $<10 \mathrm{~min}$ in most cases and the entire procedure requires minimal input from the operator.

In the case presented herein, the $\mathrm{FFR}_{\text {angio }}$ system detected the two lesions seen in the $2 \mathrm{D}$ angiography which were evaluated with invasive FFR and successfully performed a lesionper-lesion evaluation of the coronary physiology, without necessitating fragmentation of the measurements. Thus, the impact of the stenoses on the coronary tree was fully presented to the operator as a physiological roadmap. With the fact that the $\mathrm{FFR}_{\text {angio }}$ system is considered an investigational device, only the invasive FFR was used to guide treatment.

While other groups have attempted to use angiographic data to simulate invasive FFR measurements, [3-6, 8, 9, 11, 12], the methods are laborious, require additional analysis, and often involve long processing times which are limited to offline analysis by a core laboratory. Of special interest is the quantitative flow ratio (QFR) method. Tu et al. used the QFR method derived from 3 different flow models (QAngio XA 3D prototype and Medis, Leiden, Netherlands) based on 3D QCA of vessel segments and the flow moving through the stenosis [8]. This system relies on 3D QCA combined with additional TIMI frame count from high-quality images (30 frames/second) for the calculation of mean volumetric flow rate at hyperemia and still requires induced hyperemic conditions. This method also provides assessment of the main vessel of interest without providing the side branches.

\section{Conclusion}

In summary, we have demonstrated for the first time a coronary case utilizing "on-line" $\mathrm{FFR}_{\text {angio }}$ technology, showing good concordance with the wire-based FFR in sequential lesions of the LAD both before and after stenting. The system allowed for comprehensive functional evaluation of the vessel without the need for drug-induced hyperemia and/or fragmentation of the measurements, as required using conventional invasive FFR. Once confirmed in larger studies and on a wide spectrum of coronary lesions, FFR $_{\text {angio }}$ should emerge as an important tool for physiologic assessment of coronary artery disease.

\section{Conflicts of Interest}

Professor Ran Kornowski is a cofounder and an equity shareholder in CathWorks Ltd.

\section{Acknowledgments}

This study was funded by CathWorks Ltd.

\section{References}

[1] E. Barbato, G. G. Toth, N. P. Johnson et al., "A Prospective Natural History Study of Coronary Atherosclerosis Using Fractional Flow Reserve," Journal of the American College of Cardiology, vol. 68, no. 21, pp. 2247-2255, 2016.

[2] A. Ntalianis, C. Trana, O. Muller et al., "Effective radiation dose, time, and contrast medium to measure fractional flow reserve," JACC: Cardiovascular Interventions, vol. 3, no. 8, pp. 821-827, 2010.

[3] P. D. Morris, D. Ryan, A. C. Morton et al., "Virtual fractional flow reserve from coronary angiography: Modeling the significance of coronary lesions. Results from the VIRTU-1 (VIRTUal fractional flow reserve from coronary angiography) study, JACC: Cardiovascular Interventions, vol. 6, no. 2, pp. 149$157,2013$.

[4] P. D. Morris, F. N. Van De Vosse, P. V. Lawford, D. R. Hose, and J. P. Gunn, "'virtual' (Computed) Fractional Flow Reserve Current Challenges and Limitations," JACC: Cardiovascular Interventions, vol. 8, no. 8, pp. 1009-1017, 2015.

[5] M. I. Papafaklis, T. Muramatsu, Y. Ishibashi et al., "Fast virtual functional assessment of intermediate coronary lesions using routine angiographic data and blood flow simulation in humans: Comparison with pressure wire - fractional flow reserve," EuroIntervention, vol. 10, no. 5, pp. 574-583, 2014.

[6] M. Tröbs, S. Achenbach, J. Röther et al., "Comparison of Fractional Flow Reserve Based on Computational Fluid Dynamics Modeling Using Coronary Angiographic Vessel Morphology Versus Invasively Measured Fractional Flow Reserve," American Journal of Cardiology, vol. 117, no. 1, pp. 29-35, 2016. 
[7] S. Tu, E. Barbato, Z. Köszegi et al., "Fractional flow reserve calculation from 3-dimensional quantitative coronary angiography and TIMI frame count: a fast computer model to quantify the functional significance of moderately obstructed coronary arteries," Journal of the American College of Cardiology, vol. 7, no. 7, pp. 768-777, 2014.

[8] S. Tu, C. V. Bourantas, B. L. Nørgaard, G. S. Kassab, B.-K. Koo, and J. H. C. Reiber, "Image-based assessment of fractional flow reserve," EuroIntervention, vol. 11, pp. V50-V54, 2015.

[9] S. Tu, J. Westra, J. Yang et al., "Diagnostic Accuracy of Fast Computational Approaches to Derive Fractional Flow Reserve From Diagnostic Coronary Angiography: The International Multicenter FAVOR Pilot Study," JACC: Cardiovascular Interventions, vol. 9, no. 19, pp. 2024-2035, 2016.

[10] R. Kornowski, I. Lavi, M. Pellicano et al., "Fractional Flow Reserve Derived From Routine Coronary Angiograms," Journal of the American College of Cardiology, vol. 68, no. 20, pp. 22352237, 2016.

[11] C. A. Taylor, T. A. Fonte, and J. K. Min, "Computational fluid dynamics applied to cardiac computed tomography for noninvasive quantification of fractional flow reserve: scientific basis," Journal of the American College of Cardiology, vol. 61, no. 22, pp. 2233-2241, 2013.

[12] S. J. Chen and J. D. Carroll, "3-D reconstruction of coronary arterial tree to optimize angiographic visualization," IEEE Transactions on Medical Imaging, vol. 19, no. 4, pp. 318-336, 2000. 


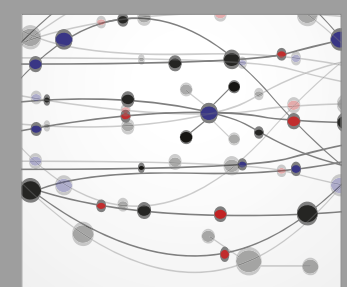

The Scientific World Journal
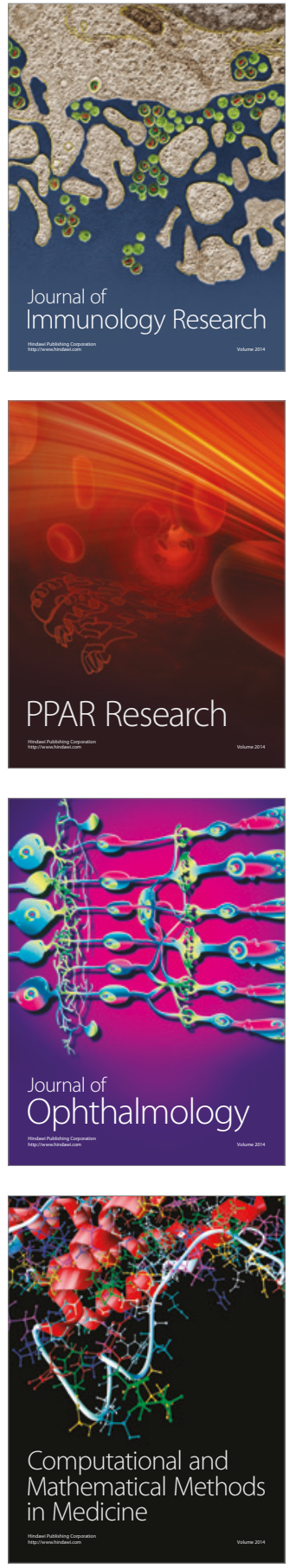

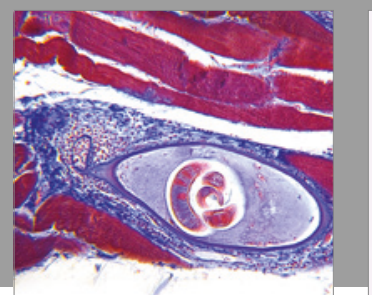

Gastroenterology Research and Practice
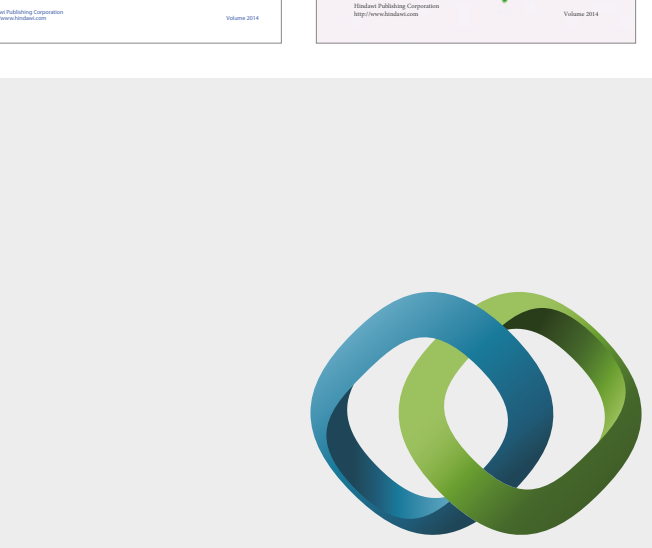

\section{Hindawi}

Submit your manuscripts at

https://www.hindawi.com
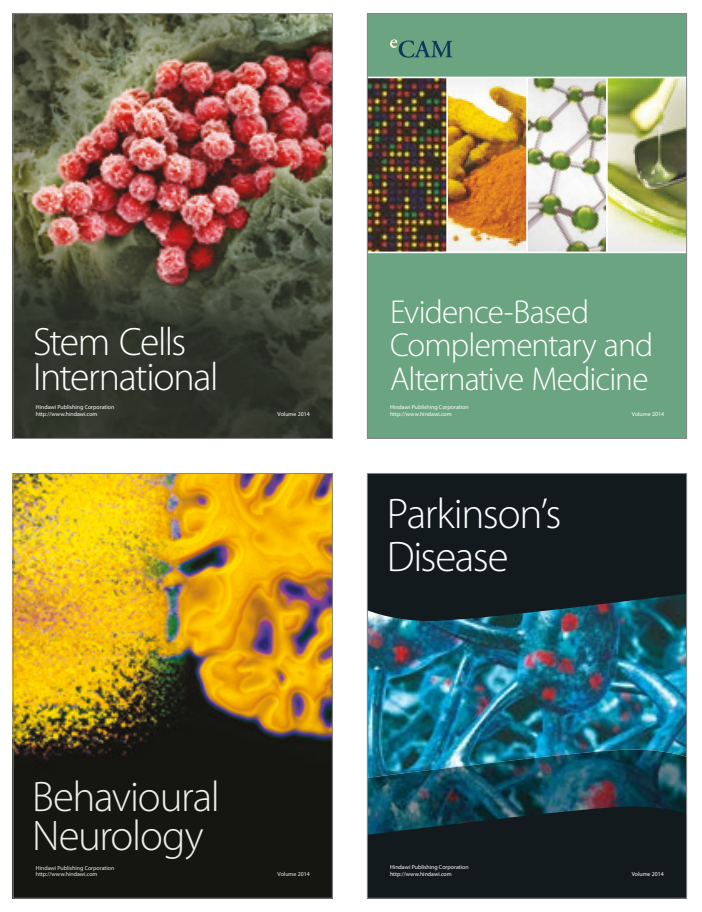
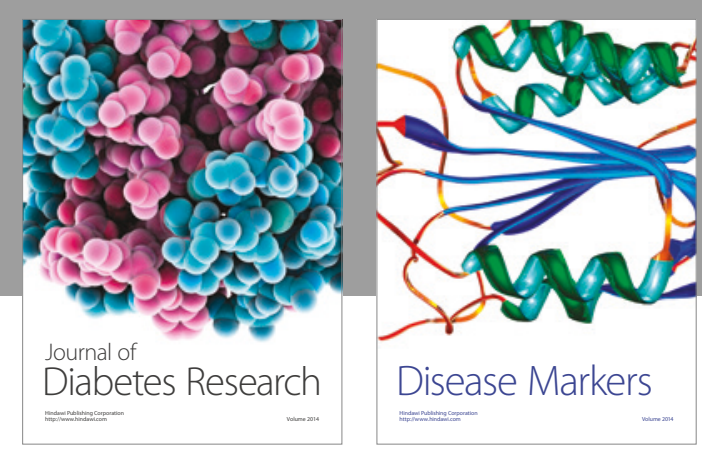

Disease Markers
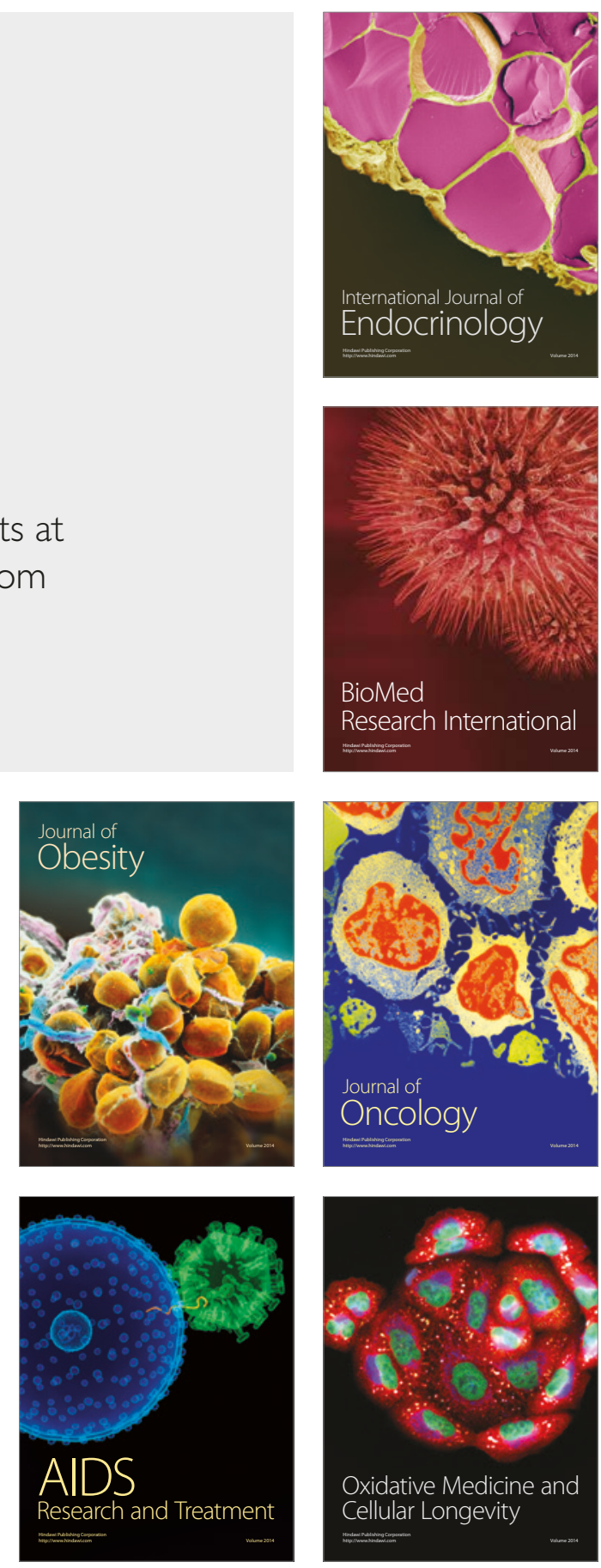